\title{
Beauty underground: cavefishes in China
}

\author{
Yahui Zhaoł, Danté B. Fenolio§, Daphne Soares', Andrew Gluesenkamp§, Matthew L. Niemillerף, \\ Maria Elina Bichuette ${ }^{\#}$, Prosanta Chakrabarty ${ }^{a}$ \\ ‡ Institute of Zoology, Chinese Academy of Sciences, Beijing, China \\ $\S$ San Antonio Zoo, San Antonio, United States of America \\ | New Jersey Institute of Technology, New Jersey, United States of America \\ I The University of Alabama in Huntsville, Huntsville, United States of America \\ \# Universidade Federal de São Carlos, São Carlos, Brazil \\ a Louisiana State Unviersity, Baton Rouge, United States of America
}

Corresponding author: Yahui Zhao (zhaoyh@ioz.ac.cn)

Received: 02 Sep 2018 | Published: 04 Sep 2018

Citation: Zhao Y, Fenolio D, Soares D, Gluesenkamp A, Niemiller M, Bichuette M, Chakrabarty P (2018) Beauty underground: cavefishes in China. ARPHA Conference Abstracts 1: e29462.

https://doi.org/10.3897/aca.1.e29462

\begin{abstract}
Cavefishes are a distinctive group of animals restricted to subterranean environments for at least for part of their lives. Their level of troglomorphism differentiates them into groups: stygobionts (troglomorphic) and stygophiles/stygoxenes (non-troglomorphic). China is home to the greatest diversity of cavefishes in the world, with more than 76 endemic stygobiotic species and an additional 70+ non-stygobiotic taxa. All but one species ( Onychostoma macrolepis in northern China), are found in the southwest, primarily in the karst of the Yunnan-Guizhou Plateau. Sinocyclocheilus, the largest cavefish genus with 35 stygobiotic species, has undergone rapid evolution and diversification. Many species in this genus possess horns and humpbacks (i.e., horn-like structures and hyperdevelopment of a dorsal protuberance similar to a humpback), probably resulting from parallel evolution. Syntopy (inhabitation of the same cave system by multiple cavefishes) is common in China, and sometimes several stygobiotic species are found in the same cave pool or subterranean river. Caves and karst habitats are fragile environments, and cavefishes are extremely sensitive to environmental change. Threats from rapid economic growth in recent decades have increased the need for conservation efforts for cave-dwelling communities, and the conservation of Chinese cavefishes is an urgent issue, in particular. To this end, the Chinese Cavefish Working Group (CCWG) was created form a collaborative effort by
\end{abstract}


researchers from China and the United States. The primary goal of the CCWG is to document the distribution and diversity of Chinese cavefishes, to gather information on their status and threats to wild populations and describe unknown aspects of their organismal biology. These data can be used to make sound conservation and management decisions. Research efforts are not limited to surveys and collection but also taxonomy, systematics, morphology, ecology, behavior, sensory neuroethology, evolution, population biology, and conservation science.

\section{Keywords}

Cavefish; Species diversity; Distribution; Conservation; China

\section{Presenting author}

Yahui Zhao

\section{Presented at}

Aveiro, Portugal

\section{Acknowledgements}

This study was conducted under the auspices of the Chinese Cavefish Working Group and supported by grants (NSFC-31471961) from the National Nature Science Foundation of China, a grant (GEFC-15-16) from National Geography Society, a grant from Ocean Park Conservation Foundation Hong Kong (P.PROJ.GEN.FH01_1819) and a grant (Y229YX5105) from the Key Laboratory of the Zoological Systematics and Evolution of the Chinese Academy of Sciences.

\section{Hosting institution}

University of Aveiro 